\title{
A generalized Grad-Shafranov equation with plasma flow under a conformal coordinate transformation
}

\author{
A. Kuiroukidis ${ }^{1}$, D. Kaltsas ${ }^{2}$ and G. N. Throumoulopoulos ${ }^{2}$ \\ ${ }^{1}$ Department of Informatics, Technological Education Institute of Serres, \\ GR 62124 Serres, Greece \\ ${ }^{2}$ Physics Department, University of Ioannina, GR 45110 Ioannina, Greece
}

Emails: kouirouki@astro.auth.gr, dkaltsas@cc.uoi.gr, gthroum@cc.uoi.gr

\begin{abstract}
We employ a conformal mapping transformation to solve a generalized Grad-Shafranov equation with incompressible plasma flow of arbitrary direction and construct particular up-down asymmetric Dshaped and diverted tokamak equilibria. The proposed method can also be employed as an alternative quasi-analytic method to solving two dimensional elliptic partial differential equations.
\end{abstract}

Two dimensional axisymmetric MHD equilibria relevant to fusion plasmas are governed by the Grad-Shafranov (GS) equation [1, a second order elliptic non-linear partial differential equation. Since plasma flow plays a role in the transition to improved confinement regimes in tokamaks, as the $\mathrm{L}-\mathrm{H}$ transition, generalized GS equations for flowing plasmas have also been obtained (e.g. Eq. (11) below). Owing to non-linearity the above mentioned equations must in general be solved numerically. One of the employed methods involves conformal mapping transformations appropriate to adapt the real shaping of the magnetic surfaces to simpler in shape ones (usually circular) in the mapped plane [2]. This mapping facilitates solving numerically the equilibrium as well as the stability problem. In addition, conformal mapping was employed to transform a linearized GS equation, obtain analytic solutions and construct compact toroidal equilibrium configurations [3]. Aim of the present note is to generalize the study [3] by employing a more generic conformal mapping transformation to solve the generalized GS equation (1), and construct configurations of tokamak relevance.

The generalized GS equation governing axisymmetric equilibria with nonparallel incompressible flow [4, 5, in normalized coordinates $(\rho, \zeta)$ can be put in the form:

$$
\Delta^{*} \psi(\rho, \zeta)=-\left[I(\psi) \frac{d I(\psi)}{d \psi}+\rho^{2} \frac{d P_{s}(\psi)}{d \psi}+\rho^{4} \frac{d G(\psi)}{d \psi}\right]
$$


Here, $\rho:=R / R_{0}, \zeta:=z / R_{0}$ where $(R, \varphi, z)$ are cylindrical coordinates and $R_{0}$ is a reference length; $\psi(\rho, \zeta)$ is the poloidal magnetic flux function;

$$
\Delta^{*}:=\frac{\partial}{\partial \rho}\left(\frac{1}{\rho} \frac{\partial}{\partial \rho}\right)+\frac{\partial^{2}}{\partial \zeta^{2}}
$$

$I(\psi), P_{s}(\psi), G(\psi)$ are freely specified functions where $I(\psi) / \rho$ is the toroidal component of the magnetic field, $P_{s}(\psi)$ is the plasma pressure in the absence of flow and $G(\psi)$ is related to the electric field and the density which is uniform on magnetic surfaces due to incompressibility. It is also noted that, owing to the flow the pressure, current and magnetic surfaces constitute there different sets of surfaces.

We will employ the conformal transformation

$$
\zeta+i \rho=g(w)=g(u+i v)=\zeta(u, v)+i f(u) \phi(v)
$$

which maps the coordinates $(\rho, \zeta, \varphi)$ in the new orthogonal system of coordinates $(u, v, \varphi)$. Using the Cauchy-Riemann conditions for the analyticity of the transformation

$$
\begin{aligned}
\frac{\partial \zeta}{\partial u} & =f(u) \phi^{\prime}(v), \quad \frac{\partial \zeta}{\partial v}=-f^{\prime}(u) \phi(v) \\
\frac{d g(w)}{d w} & =\frac{\partial \zeta}{\partial u}+i f^{\prime}(u) \phi(v)=f(u) \phi^{\prime}(v)+i f^{\prime}(u) \phi
\end{aligned}
$$

the operator (2) is transformed into

$$
\tilde{\Delta}^{*}=h^{2} \rho(u, v)\left[\frac{\partial}{\partial u}\left(\frac{1}{\rho(u, v)} \frac{\partial}{\partial u}\right)+\frac{\partial}{\partial v}\left(\frac{1}{\rho(u, v)} \frac{\partial}{\partial v}\right)\right]
$$

where $1 / h^{2}:=|d g(w) / d w|^{2}$. In order to solve Eq. (1) by the method of separation of variables we now adopt the linearing ansatz

$$
\frac{d P(\psi)}{d \psi}=b, \quad I(\psi) \frac{d I(\psi)}{d \psi}=A^{2} \psi+\kappa, \quad \frac{d G(\psi)}{d \psi}=G_{0}
$$

where $b, A^{2}, \kappa, G_{0}$ are non-zero parameters. Then we also assume separability of the following functions

$$
\psi_{h}(u, v)=L(u) M(v), \quad \rho(u, v)=f(u) \phi(v)
$$


where $\psi_{h}$ is the general solution to the homogeneous part of Eq. (1). Using (7) Eq. (1) becomes

$$
\frac{f}{L} \frac{d}{d u}\left(\frac{1}{f} \frac{d L}{d u}\right)+\frac{\phi}{M} \frac{d}{d v}\left(\frac{1}{\phi} \frac{d M}{d v}\right)+A^{2}\left[f^{2}\left(\phi^{\prime}\right)^{2}+\left(f^{\prime}\right)^{2} \phi^{2}\right]=0
$$

We now generalize the analysis of [3] by using Eqs. (44) and making the choice

$$
\begin{aligned}
f & :=f_{1} \cos (k u)+f_{2} \sin (k u) \\
\phi & :=\phi_{1} \cosh (k v)+\phi_{2} \sinh (k v)
\end{aligned}
$$

with the further definitions $\xi:=\cos (k u), \eta:=\cosh (k v),(0 \leq \xi \leq 1),(\eta \geq$ $1)$. The choice (9) includes the prolate and oblate spheroidal systems of coordinates employed in [3] as particular cases. Here $f_{1}, f_{2}, \phi_{1}, \phi_{2}, k$ are arbitrary non-zero parameters. Then from Eqs. (4) we obtain

$$
\begin{aligned}
\zeta=\left[f_{1} \sin (k u)\right. & \left.-f_{2} \cos (k u)\right]\left[\phi_{1} \sinh (k v)+\phi_{2} \cosh (k v)\right] \\
f(\xi) & =f_{1} \xi+f_{2} \sqrt{1-\xi^{2}} \\
\phi(\eta) & =\phi_{1} \eta+\phi_{2} \sqrt{\eta^{2}-1}
\end{aligned}
$$

Furthermore, we use (6) into the generalized GS Eq. (11) by writing its solution as $\psi=\psi_{p}+\psi_{h}$, that is as a superposition of a particular solution $\psi_{p}$ of the inhomogeneous Eq. (8) plus a general solution $\psi_{h}$ of the respective homogeneous equation; the particular solution is

$$
\psi_{p}=\frac{1}{A^{2}}\left(-b+\frac{8 G_{0}}{A^{2}}\right)(f(\xi) \phi(\eta))^{2}-\frac{G_{0}}{A^{2}}(f(\xi) \phi(\eta))^{4}-\frac{\kappa}{A^{2}}
$$

The homogeneous equation leads to the following ODEs for $L(\xi)$ and $M(\eta)$ :

$$
\begin{array}{r}
\left(1-\xi^{2}\right) \frac{d^{2} L(\xi)}{d \xi^{2}}+f(\xi) \sqrt{1-\xi^{2}} \frac{d}{d \xi}\left[\frac{\sqrt{1-\xi^{2}}}{f(\xi)}\right] \frac{d L(\xi)}{d \xi} \\
+\left[\frac{\Lambda^{2}}{k^{2}}-A^{2}\left(f_{1}^{2}+f_{2}^{2}\right)\left(\phi_{1}^{2}+\phi_{2}^{2}\right) \xi^{2}-2 A^{2} f_{1} f_{2}\left(\phi_{1}^{2}-\phi_{2}^{2}\right) \xi \sqrt{1-\xi^{2}}\right] L(\xi)=0 \\
\left(\eta^{2}-1\right) \frac{d^{2} M(\eta)}{d \eta^{2}}+\phi(\eta) \sqrt{\eta^{2}-1} \frac{d}{d \eta}\left[\frac{\sqrt{\eta^{2}-1}}{\phi(\eta)}\right] \frac{d M(\eta)}{d \eta} \\
+\left[-\frac{\Lambda^{2}}{k^{2}}+A^{2}\left(f_{1}^{2}+f_{2}^{2}\right)\left(\phi_{1}^{2}+\phi_{2}^{2}\right) \eta^{2}-2 A^{2} \phi_{1} \phi_{2}\left(f_{1}^{2}+f_{2}^{2}\right) \eta \sqrt{\eta^{2}-1}\right] M(\eta)=0
\end{array}
$$


where $\Lambda$ is the separability constant for Eq. (8).

We have solved numerically Eqs. (14) using the fourth-order Runge-Kutta method for the intervals $0 \leq \xi \leq 1,1 \leq \eta \leq 4$ with step size $1 / N, N=125$. As a concrete, specific example, the various constants were taken to have the following values: $k=2 \pi / 1.92, A=1.95, \Lambda=1.5, f_{0}=0.2825, f_{1}=$ $\sqrt{13} f_{0} / 7, f_{2}=\sqrt{3} f_{1}, \phi_{0}=2.4, \phi_{1}=1.05, \phi_{2}=\phi_{0} \phi_{1}=2.52, b=0.0195$, $G_{0}=0.145, \kappa=0.05$. The initial conditions were taken to be $L(\xi=0)=1.0$, $L^{\prime}(\xi=0)=0.1, M(\eta=1)=1.0, M^{\prime}(\eta=1)=0.1$. This results in the functions $L(\xi), M(\eta)$, shown in Fig. 1 and in the up-down asymmetric Dshaped equilibrium shown in Fig. 2. It is noted that up-down asymmetry may drive fast intrinsic rotation in tokamaks [6]. The bounding flux surface, shown in blue corresponds to $\psi_{b}=-1.49$ while on the magnetic axis, also shown in blue, we have $\psi_{a}=-2.15$. The magnetic axis is located at the point $\left(\rho_{a}, \zeta_{a}\right)=(2.095,0.09)$. Its elongation is $K=2.4627$, while its triangularity is $\delta=0.83$. This equilibrium has peaked on the magnetic axis pressure and toroidal current density profiles shown in Fig. 3.

We also have found numerically that the separability constant $\Lambda$ can be "quantized", in the sense that for an infinite set of discrete values $\Lambda_{n}, \quad(n=$ $1,2, \ldots)$, the corresponding solutions $L_{n}(\xi)$ of Eq. (14a) are mutually orthogonal, i.e.

$$
I_{n, m}:=\int_{0}^{1} d \xi L_{n}(\xi) L_{m}(\xi)=0, \quad(n \neq m)
$$

A similar orthogonalization can be made for the solutions $M_{n}(\eta)$ of Eq. (14 b). We have verified numerically that this quantization holds for broad regions of the free parameters $f_{1}, f_{2}, \phi_{1}, \phi_{2}, k, A, b, G_{0}, \kappa$. So a more generic solution of Eq. (1) can be written as a superposition of these mutually orthogonal functions of Eqs. (14):

$$
\tilde{\psi}(\rho, \zeta)=\psi(\xi(\rho, \zeta), \eta(\rho, \zeta))=\psi_{p}+\sum_{n=1}^{\infty} D_{n} L_{n}(\xi) M_{n}(\eta)
$$

where $\psi_{p}$ is given by Eq. (13), $\xi$ and $\eta$ can be expressed in terms of $\rho$ and $\zeta$ by means of $\xi=\cos (k u), \eta=\cosh (k v)$, (3) and (9); $D_{n}$ are arbitrary constants. As a concrete example for the parametric values and initial conditions given above in the previous paragraph we have found numerically that for the following choice for the "quantized" separability constant

$$
\Lambda_{n}=n^{d}+c, \quad(n=1,2, \ldots), \quad d=1.6, \quad c=1.81
$$


the orthogonality condition of Eq. (15) holds true, numerically, to a high degree of precision. We conjecture that this holds true for any values of the free parameters and the initial conditions involved. Thus, Eq. (16) can be employed to construct more generic equilibria. A particular diverted equilibrium with a lower X-point located at the low field side is given in Fig. 4. This equilibrium corresponds to the first value of the parameter $\Lambda_{1}=3.41$ of Eq. (17) with a single non vanishing term in the sum of Eq. (16) with $D_{1}=1$. According to experimental results in the TCV tokamak, the radial position of the $\mathrm{X}$-point relates to edge intrinsic toroidal rotation in correlation with the core rotation [7].
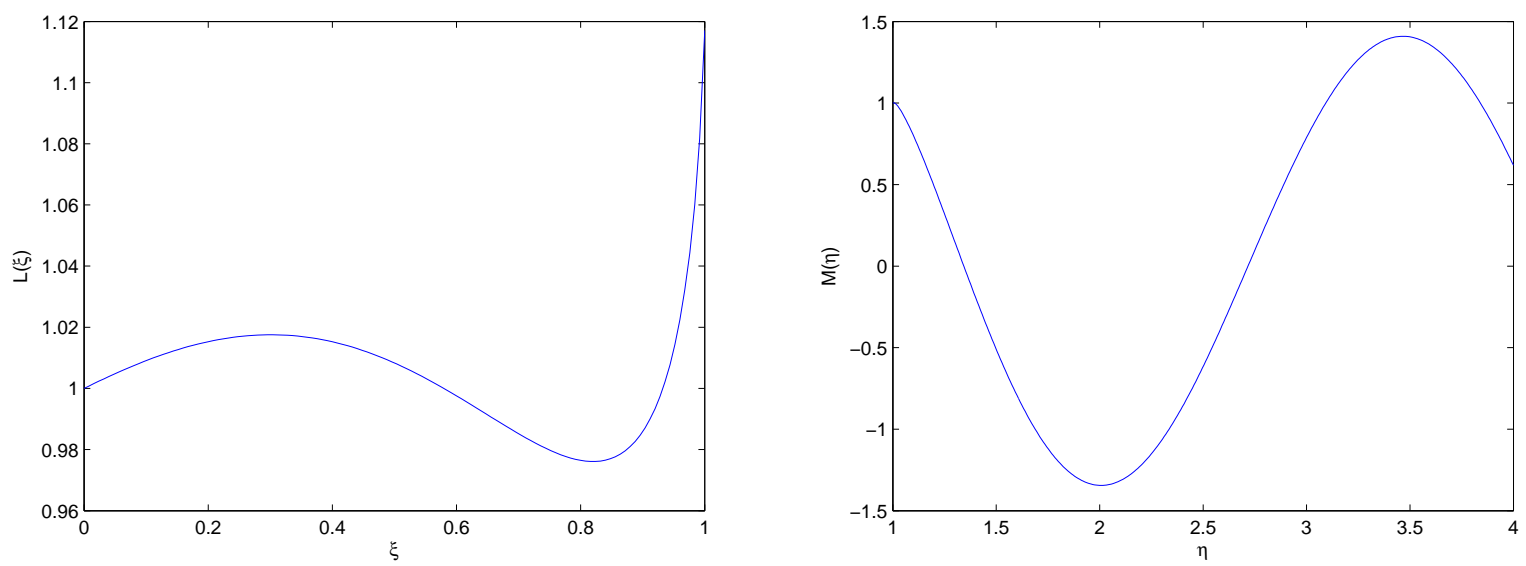

Figure 1: The functions $L(\xi)$ and $M(\eta)$ as solutions of Eqs. (14a) and (14b) for initial conditions and parametric values as described in the text of the fourth paragraph.

Summarizing, we have employed a conformal mapping transformation to solve a generalized GS equation with incompressible flow of arbitrary direction by the method of separation of variables. Appropriate choices of the mapping function permits the construction of configurations with desirable shaping. As examples particular up-down asymmetric tokamak pertinent equilibria either with D-shaped magnetic surfaces or a single X-point were constructed.

We end up with a couple of remarks in connection with potential extension and improvement of the present study. In the development of HELENA code [8, 9], which solves the GS equation for a plasma surrounded by a fixed pre- 


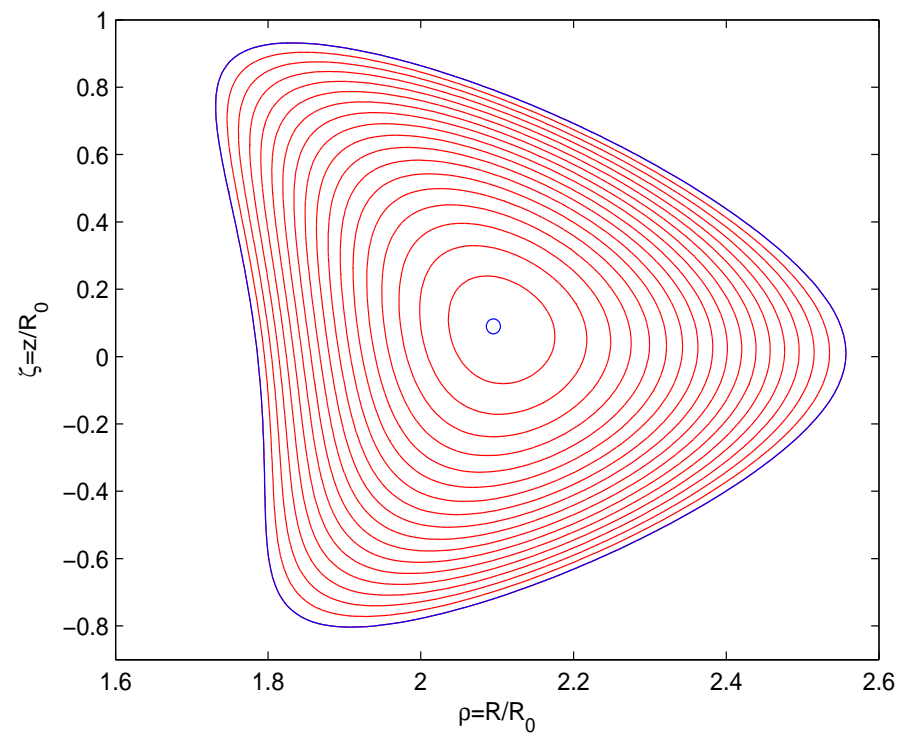

Figure 2: The equilibrium obtained by numerical solutions of Eqs. (14) associated with the homogeneous GS equation (8) and the analytic special solution (13) of the respective inhomogeneous equation.

scribed boundary, it was realized that the conformal coordinates produced a quite poor angular distribution of the grid. This drawback dictated the employment of particular finite element coordinates instead of the conformal ones to map the magnetic surfaces to a set of concentric circles. The same mapping was employed to extend the above mentioned code by including pressure anisotropy and toroidal flow [10] and flow parallel to the magnetic field [11]. Also, this approach was suitable for the stability studies with CASTOR 12, the later development of the FINESSE equilibrium code for compressible flow [13] and the associated spectral code PHOENIX [14]. Second, the method of expansion functions of Eq. (16) could be adopted into a more general setting, which transcends the assumption of linear profiles for the free equilibrium functions and the separability of the final equation adopted here, in line with previous work on the subject [15], which is also appropriate for stability considerations. This kind of expansion relies on a conformal mapping of the computational domain on the unit circle using Hilbert transform with simultaneous relocation of the magnetic axis in the centre of the circle upon employing a Moebius transform as in [2]. Such 

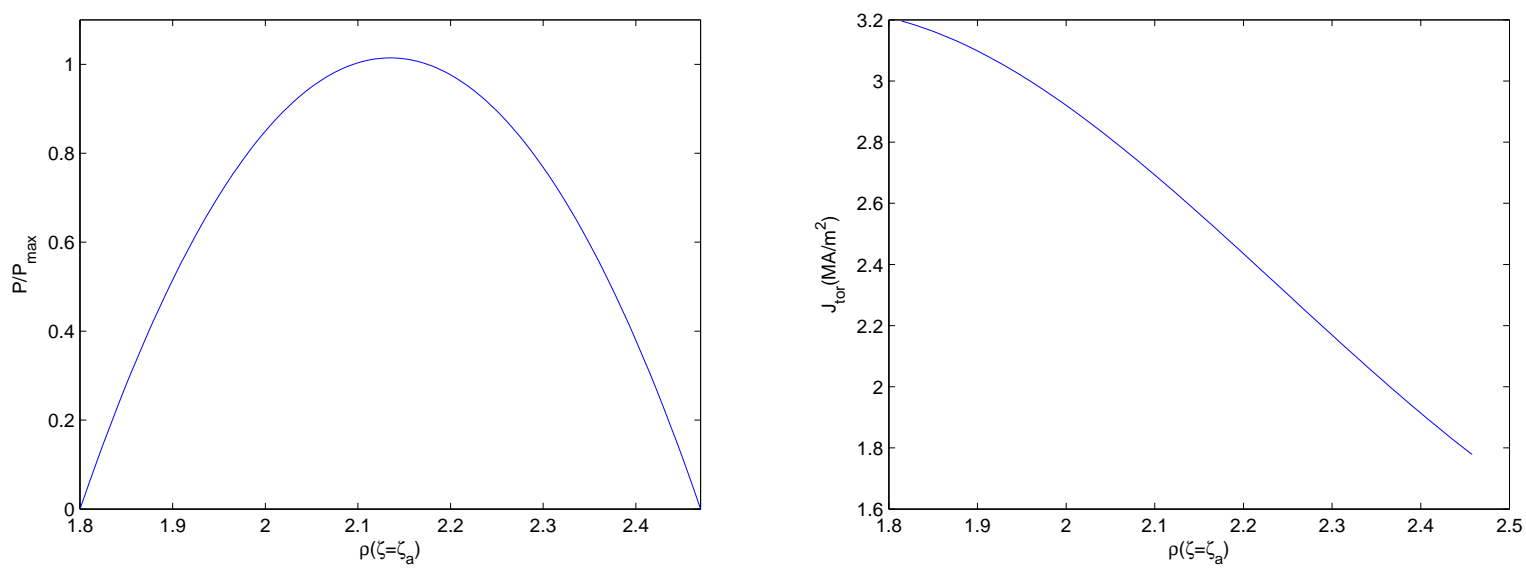

Figure 3: Profiles of the pressure and current density for the equilibrium of Fig. 2, on the horizontal line passing through the magnetic axis located at $\zeta=\zeta_{a}=0.21$. The pressure has been normalized with respect to $P_{0}=1$ atm.

an approach could potentially be adopted in order to solve the generalized Grad-Shafranov equation (11). We aim to investigate this possibility in a future work.

\section{Aknowledgments}

This study was performed within the framework of the EUROfusion Consortium and has received funding from the National Program for the Controlled Thermonuclear Fusion, Hellenic Republic. The views and opinions expressed herein do not necessarily reflect those of the European Commission. D.A.K. was supported by a Ph.D grant from the Hellenic Foundation for Research and Innovation (HFRI) and the General Secretariat for Research and Technology (GSRT). The authors would like to acknowledge the anonymous Reviewer for critical comments that helped to improve the paper. 


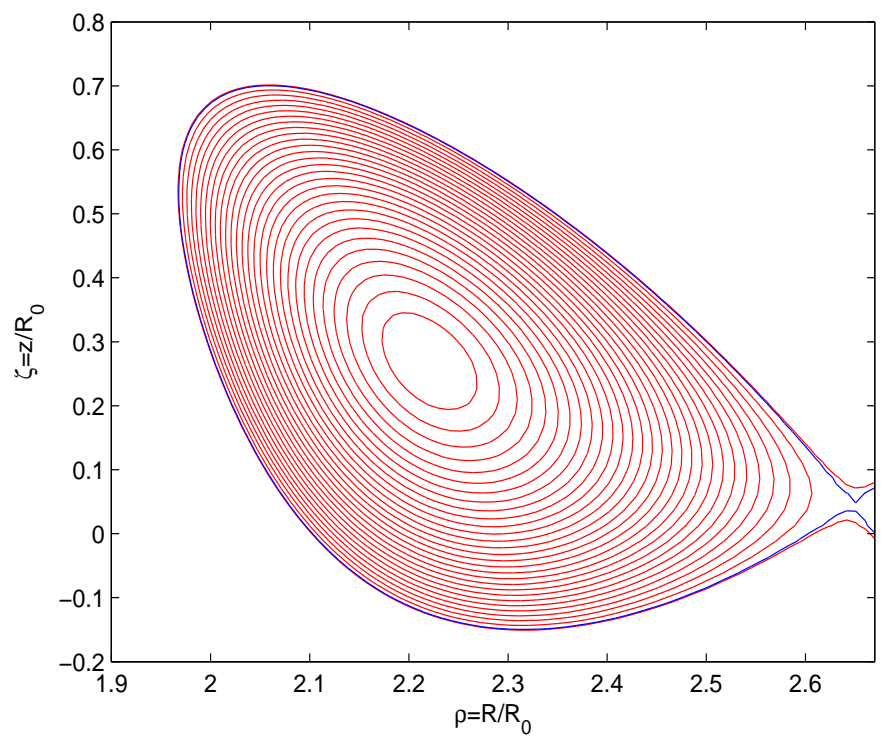

Figure 4: The solution of Eq. (16) for $D_{1}=1$ and $D_{n}=0$ for $n \neq 1$ corresponding to the first discrete value $\Lambda_{1}=3.41$ of Eq. (17) in connection with the orthogonality relation (15).

\section{References}

[1] J. P. Freidberg, Rev. Mod. Phys. 54 (1982) 801.

[2] J. P. Goedbloed, Phys. Fluids, 25 (1982) 2073; Computer Physics Communications 31, 123 (1984); Physica 12D, 107 (1984) .

[3] G. N. Throumoulopoulos and G. Pantis, Nucl. Fusion 26, No. 111501 (1986).

[4] H. Tasso and G. N. Throumoulopoulos, Phys. Plasmas 5, 2378 (1998).

[5] Ch. Simintzis, G. N. Throumoulopoulos, G. Pantis, and H. Tasso, Phys. Plasmas 8, 2641 (2001).

[6] J. Ball, F. I. Parra, M. Landreman and M. 1. Barnes, Nucl. Fusion 58, 026003 (2018).

[7] T. Stoltzfus-Dueck, Y. Camenen, PRL 114, 245001 (2015). 
[8] T. A. Huysmans, J. P. Goedbloed, and W. Kerner, Int. J. Mod. Phys. C 2(01), 371 (1991).

[9] C. Konz and R. Zille, Manual of HELENA Fixed Boundary Equilibrium Solver (Max-Planck Institute for Plasma Physics, 2007).

[10] Z. S. Qu, M. Fitzgerald and M. J. Hole, Plasma Phys. Control. Fusion 56, 075007 (2014).

[11] G. Poulipoulis, G. N. Throumoulopoulos, C. Konz, and ITM-TF Contributors, Phys. Plasmas 23, 072507 (2016).

[12] W. Kerner, J. P. Goedbloed, G. T. A. Huysmans, S. Poedts, E. Schwarz, J. Comp. Phys. 142, 271 (1998).

[13] A. J. C. Beliën, M. A. Botchev, J. P. Goedbloed, B. van der Holst, R. Keppens, J. Comp. Phys. 182, 91 (2002).

[14] J. W. S. Blokland, B. van der Holst, R. Keppens, J. P. Goedbloed, J. Comp. Phys. 226, 509 (2007).

[15] J. P. Goedbloed, J. Comp. Phys. 160, 283 (2000). 